OPEN ACCESS

Edited by:

Lavinia Alberi,

University of Fribourg, Switzerland

Reviewed by:

Hermona Soreq,

Hebrew University of Jerusalem, Israe Adriano Barreto Nogueira,

University of São Paulo, Brazil

*Correspondence:

Tobias Bobinger

Tobias.Bobinger@uk-erlangen.de

Received: 22 November 2016 Accepted: 03 March 2017

Published: 27 March 2017

Citation:

Bobinger T, May L, Lücking $H$, Kloska SP, Burkardt P, Spitzer P, Maler JM, Corbeil D and Huttner HB

(2017) CD133-Positive Membrane

Particles in Cerebrospinal Fluid of Patients with Inflammatory and Degenerative Neurological Diseases.

Front. Cell. Neurosci. 11:77. doi: $10.3389 /$ fncel.2017.00077

\section{CD133-Positive Membrane Particles in Cerebrospinal Fluid of Patients with Inflammatory and Degenerative Neurological Diseases}

\author{
Tobias Bobinger $^{1 *}$, Lisa May ${ }^{1}$, Hannes Lücking ${ }^{2}$, Stephan P. Kloska ${ }^{2}$, Petra Burkardt ${ }^{1}$, \\ Philipp Spitzer ${ }^{3}$, Juan M. Maler ${ }^{3}$, Denis Corbeil ${ }^{4}$ and Hagen B. Huttner ${ }^{1}$

\footnotetext{
${ }^{1}$ Department of Neurology, University Hospital Erlangen, Erlangen, Germany, ${ }^{2}$ Department of Neuroradiology, University Hospital Erlangen, Erlangen, Germany, ${ }^{3}$ Department of Psychiatry, University Hospital Erlangen, Erlangen, Germany,

${ }^{4}$ Biotechnology Center, Technische Universität Dresden, Dresden, Germany
}

Background: Analysis of cerebrospinal fluid (CSF) is a frequently used diagnostic tool in a variety of neurological diseases. Recent studies suggested that investigating membrane particles enriched with the stem cell marker CD133 may offer new avenues for studying neurological disease. In this study, we evaluated the amount of membrane particle-associated CD133 in human CSF in neuroinflammatory and degenerative diseases.

Methods: We compared the amount of membrane particle-associated CD133 in CSF samples collected from 45 patients with normal pressure hydrocephalus, parkinsonism, dementia, and cognitive impairment, chronic inflammatory diseases and 10 healthy adult individuals as controls. After ultracentrifugation of CSF, gel electrophoresis and immunoblotting using anti-CD133 monoclonal antibody 80B258 were performed. Antigen-antibody complexes were detected using chemiluminescence.

Results: The amount of membrane particle-associated CD133 was significantly increased in patients with normal pressure hydrocephalus $(p<0.001)$, parkinsonism $(p=0.011)$ as well as in patients with chronic inflammatory disease $(p=0.008)$. Analysis of CSF of patients with dementia and cognitive impairment revealed no significant change compared with healthy individuals. Furthermore, subgroup analysis of patients with chronic inflammatory diseases demonstrated significantly elevated levels in individuals with relapsing-remitting multiple sclerosis $(p=0.023)$ and secondary progressive multiple sclerosis (SPMS; $p=0.010$ ).

Conclusion: Collectively, our study revealed elevated levels of membrane particleassociated CD133 in patients with normal pressure hydrocephalus, parkinsonism as well as relapsing-remitting and SPMS. Membrane glycoprotein CD133 may be of clinical value for several neurological diseases.

Keywords: CD133, cerebro-spinal fluid, multiple sclerosis, membrane particles, neurodegenerative disorders 


\section{INTRODUCTION}

The cerebrospinal fluid (CSF) is frequently analyzed for diagnosing a variety of neurological diseases. The basic CSFanalysis includes cell count, cytological findings and total amount of protein (Johanson et al., 2011). Specific clinical CSF-analyses focus on protein-based alterations such as oligoclonal banding, particular antibodies, or particular soluble proteins such as tau (Hühmer et al., 2006). Yet, CSF may have a far greater diagnostic potential considering recent studies demonstrating that CSF contains small membrane particles (Huttner et al., 2008, 2012; Colombo et al., 2012; Chiasserini et al., 2014).

As previously shown, CSF-associated membrane particles may contain CD133, a cholesterol-binding pentaspan membrane glycoprotein (Figure 1). Although the biological function of this glycoprotein remains to be demonstrated, it gained attention in the past few years. CD133 labels stem and progenitor cells in various organs notably in the neural and hematopoietic system (Miraglia et al., 1997; Weigmann et al., 1997; Jászai et al., 2013; Walker et al., 2013; Pruszak, 2015). CD133 highlights cancer stem cells and marks potential facultative stem cells, which might be activated during regeneration (Grosse-Gehling et al., 2013; Corbeil et al., 2014). Besides its expression in progenitor cells, CD133 is detected in differentiated cells such as epithelial and glial cells (Karbanová et al., 2008; Corbeil et al., 2009). In the adult brain it is expressed on multiciliated ependymal cells and subventricular zone astrocytes. Therein, CD133 is concentrated in ependymal cilia as well as in primary cilia of quiescent neural stem cells that are exposed to CSF (Dubreuil et al., 2007; Coskun et al., 2008; Huttner et al., 2008; Codega et al., 2014; Karbanová et al., 2014; Nogueira et al., 2014). CD133 is also detected in myelin sheaths of oligodendrocytes and Schwann cells found

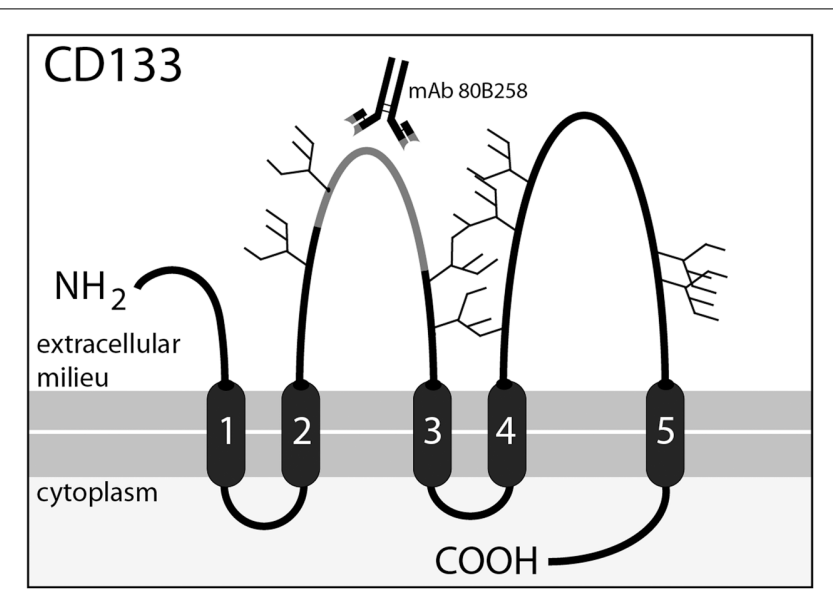

FIGURE 1 | Membrane topology of CD133 and location of the 80B258 epitope. CD133 consists of an extracellular $\mathrm{N}$-terminal domain, 5-transmembrane domains separating two large glycosylated extracellular loops and two small intracellular loops, and an intracellular C-terminal domain. Potential N-glycosylation sites are indicated with forks. Mouse-mAb 80B258 was generated against a fusion protein containing part of the first extracellular loop (residue glycine 240 to serine 388, gray segment) of human prominin-1 (Karbanová et al., 2008). in central and peripheral nervous system, respectively (Corbeil et al., 2009). A remarkable biological feature of CD133 is its release in several body fluids in association with membrane particles, initially referred to as "prominosomes" (Marzesco et al., 2005). Thus, CD133 can be recovered upon ultracentrifugation in urine, tear, saliva, seminal fluid and CSF (Marzesco et al., 2005; Huttner et al., 2008, 2012; Karbanová et al., 2008, 2014). Although the release of CD133-associated membrane particles can occur at a constant rate, particularly in healthy epithelial cells contacting a given body fluid (e.g., urine, CSF), an increase of CD133 secretion was linked to the differentiation of stem and progenitor cells (Marzesco et al., 2005; Dubreuil et al., 2007; Bauer et al., 2011). The release of CD133 in CSF can occur by the budding of membrane particles (i.e., ectosomes or microvesicles) from plasma membrane protrusions (e.g., cilia) of ependymal cells and/or underlying subventricular astrocytes (Marzesco et al., 2005; Dubreuil et al., 2007; Corbeil et al., 2009, 2013; Kriegstein and Alvarez-Buylla, 2009). The release of CD133 in association with exosomes, i.e., the internal membrane vesicles of multivesicular bodies that are discharged into the extracellular space by exocytosis (Fevrier and Raposo, 2004) cannot be excluded, particularly in cancerous cells. However, the detection of CD133 in exosomes was reported only with hematopoietic progenitors (Bauer et al., 2011).

Monitoring the amount of membrane particle-associated CD133 in body-fluids may provide information about biological activity of contacting cells and its variation in onset and progression of several diseases. To date, only two reports were published on membrane particle-associated CD133 in human CSF. In essence, these studies found an elevation of CD133 in early stage glioblastoma as well as in temporal lobe epilepsy (Huttner et al., 2008, 2012). In the light of such observations, we investigated the amount of membrane particle-associated CD133 in CSF of patients with inflammatory and degenerative neurological diseases and assessed the possible clinical value of membrane-bound CD133.

\section{MATERIALS AND METHODS}

\section{Study Design, Patient Selection and Retrieval of Clinical Data}

The study included patients admitted to the Department of Neurology, University Hospital Erlangen (Germany) and was approved by the local institutional review board. CSF samples were collected in inpatients who received a diagnostic lumbar puncture, indicated to exclude or verify neurological disease, and consented for research-based CSF analysis.

We enrolled (i) 10 patients who received CSF analysis to exclude subarachnoid hemorrhage or inflammatory disease showing normal CSF analysis and these patients are in the following referred to as healthy subjects, (ii) patients with normal pressure hydrocephalus $(n=6)$, parkinsonism $(n=6)$, dementia and cognitive impairment $(n=8)$ and chronic inflammatory central nervous system disease $(n=25)$. Clinical data and medical information were collected from our institutional 
database. In patients with chronic inflammatory central nervous system disease, the Expanded Disability Status Scale (EDSS) score was assessed during the stay (Kurtzke, 1983), the disease course was classified as clinical isolated syndrome (CIS) / relapsing remitting $(\mathrm{RR})$, secondary progressive $(\mathrm{SP})$ or primary progressive (PP) multiple sclerosis and myelitis (Rovira et al., 2009; Montalban et al., 2010). For diagnosis of multiple sclerosis, brain images were acquired. Images were done at a 1.5-T MRI (Siemens AG, Erlangen, Germany) consisting of an axial T1and T2-weighted spin echo sequences. Brain atrophy (Bermel and Bakshi, 2006) and lesion load measurements were scored as recently described (Ormerod et al., 1987; Petzold et al., 2016). Samples of patients with inflammatory diseases were obtained during patient's hospitalization for relapse therapy. In patients with dementia or cognitive impairment, the total tau protein, phospho-tau and the 42 and 40 amino acid form of amyloid $\beta$ were analyzed (Zetterberg, 2015).

\section{Isolation of CD133-Membrane Particles}

Upon lumbar puncture, an aliquot $(1 \mathrm{ml})$ from the collected CSF was frozen at $-80^{\circ} \mathrm{C}$ immediately after adding protease inhibitor according to the manufacturer's instruction (Roche Diagnostics, Cat. No. 04693116001, Mannheim, Germany). CSF samples (1 ml each) were thawed on ice and sequentially centrifuged for $30 \mathrm{~min}$ at $10.000 \times g$ at $4^{\circ} \mathrm{C}$, the resulting supernatants were collected and centrifuged for $1 \mathrm{~h}$ at $100.000 \times g$ at $4^{\circ} \mathrm{C}$. Supernatants were discarded, and $100.000 \times g$ pellets were resuspended in $35 \mu \mathrm{l}$ Laemmli buffer and analyzed by immunoblotting (see below) (Huttner et al., 2008). Extracts from human Caco-2 cells were used as positive controls and to establish the standard curve since these cells express CD133 (Corbeil et al., 2000; Marzesco et al., 2005; Huttner et al., 2008). Caco-2 cells were produced in a medium with $20 \%$ fetal calf serum, $2 \mathrm{mM}$ L-glutamine, $1 \%$ non-essential amino acids, $100 \mathrm{U} / \mathrm{ml}$ penicillin, and $100 \mu \mathrm{g} / \mathrm{ml}$ streptomycin under a $5 \% \mathrm{CO}_{2}$ atmosphere at $37^{\circ} \mathrm{C}$. Caco- 2 cells were grown until 10 days post-confluence. Cells extracts were prepared as described (Huttner et al., 2008) and the protein concentration was measured using BCA Protein Assay (BCA Protein Assay Kit Pierce, Thermo Fisher, Cat. No. 23252, Waltham, MA, USA). The Caco-2 cell extract used had a protein concentration of $10 \mu \mathrm{g} / \mu \mathrm{l}$ and was diluted 1:100.

\section{Quantitative Immunoblotting}

Probes and positive controls were subjected to Tris-Glycine gels (Precise 8\% Tris-Glycine Gels, ThermoFisher, Cat. No. 25260, Waltham, MA, USA) and transferred to polyvinylidene difluoride membranes (Immobilon-P membrane, Merck Millipore, IPVH00010, $0.45 \mu \mathrm{m}$, Billerica, MA, USA) as published (Huttner et al., 2008, 2012). After blocking for $1 \mathrm{~h}$ in PBS containing 0.3\% Tween-20 and 5\% low-fat milk powder (blocking solution), membranes were incubated for $12 \mathrm{~h}$ in blocking solution containing mouse monoclonal antibody (mAb) 80B258 (1 $\mu \mathrm{g} / \mathrm{ml}$; isotype IgG1) (Karbanová et al., 2008). Membranes were then washed thrice for $15 \mathrm{~min}$ in PBS containing 0.3\% Tween-20 and incubated for $1 \mathrm{~h}$ in blocking solution containing horseradish peroxidase conjugated goat anti-mouse secondary antibody (1:5000; Jackson Immunoresearch Laboratories,
Cat. No. 115-035-068, West Grove, PA, USA). The antigenantibody complexes were detected using Chemiluminescence reagents (SuperSignal West Femto Substrate, ThermoFisher Scientific, Cat. No. 34095, Waltham, Massachusetts, USA). Total transferred proteins were detected using Ponceau staining (Ponceau S solution, Sigma-Aldrich, Cat. No. P7170, St. Louis, MO, USA) (Supplementary Figures 1, 2). For visualization of antigen-antibody complexes, the image reader Fusion FX7 Spectra (Vilber Lourmat GmbH, Eberhardzell, Germany) was utilized, and quantification was performed using Image Quant TL (GE Healthcare Europe GmbH, Freiburg, Germany). Absorbance values within the linear range were used for statistical analysis as described (Huttner et al., 2012). An internal standard sample was established using the standard curve derived from Caco-2 cell extract and evaluated on each blot (Supplementary Figure 2). The absorbance levels of analyzed CSF samples of diseased individuals were compared to the mean absorbance level of healthy individuals and expressed as a deviation in percentages (CD133-deviation).

\section{Statistical Analysis}

Data were processed using Office 2013 software package (Microsoft Corp., Redmond, WA, USA). Statistical analysis was performed with SPSS statistical software package 21.0 (SPSS Inc., Chicago, IL, USA). Distribution of the data was established using the Kolmogorov-Smirnov test. Normal distributed data are presented as mean \pm standard deviation (SD) as well as standard error of the mean (SEM) (compared using Student's $t$-test). Other data are shown as median and interquartile ranges (compared using Mann-Whitney $U$-test). Absorbance values of each group are calculated referring to healthy subjects. A logistic regression model was used to investigate the relationship between CD133deviation and basic CSF analysis as well as EDSS-score and lesion load. All statistical tests were two-sided with a significance level of $\alpha=0.05$ without adjustment for multiple comparisons.

\section{RESULTS}

\section{Detection of CD133-Positive Membrane Particles in the CSF of Healthy and Diseased Patients}

The amount of membrane particle-associated CD133 in the CSF of healthy subjects was determined upon differential centrifugation of CSF followed by semi-quantitative immunoblotting of the resulting fractions. The CD133 glycoprotein was detected using our characterized mouse mAb 80B258, which recognized a polypeptide epitope located in its extracellular loop (Figure 1) (Karbanová et al., 2008). The blotting was performed by analyzing three defined amounts of a CD133-containing Caco-2-cell extract (for technical details and the establishment of standard curve see Materials and Methods, Supplementary Figures 1, 2). Such controls were used to establish an internal reference. From $1 \mathrm{ml}$ aliquot of CSF, we recovered $1.319 \times 10^{3} \pm 682 \times 10^{3} \mathrm{pixel} /$ absorbance units of CD133 immunoreactivity in healthy subjects. This amount was used as a 
reference to healthy subjects to determine the variation of CD133 in CSF of diseased patients.

The characteristics of all included patients with normal pressure hydrocephalus, Parkinson's disease, dementia and cognitive impairment, and chronic inflammatory disease are summarized in Tables 1, 2. Basic parameters of CSF-analysis is provided in the Supplementary Table 1. Interestingly, there was a significant increase of CD133 in CSF collected from six patients with normal pressure hydrocephalus $[261 \% \pm 81.5 \%$ $(S D)$ or $42 \%$ (SEM); $p<0.001$ ] and six others with parkinsonism $[230 \% \pm 69 \%(S D)$ or $53 \% ; p=0.011]$. In the latter cases, the increase of CD133 was observed in patients with atypical parkinsonism [two patients; $357 \% \pm 90 \%(S D)$ or $54 \%$ (SEM); $p=0.03$ ], patients with parkinson disease showed only a trend toward increased CD133 [four patients; $167 \% \pm 60 \%(S D)$ or $30 \%$ (SEM); $p=0.076]$. CSF-associated CD133 levels were also elevated in eight patients with dementia/cognitive impairment $[190 \% \pm 150 \%(S D)$ or $52 \%(S E M)]$ but the increase is not significant by comparison to healthy subjects $(p=0.237)$. Lastly, the analysis of 25 patients with chronic inflammatory diseases revealed an elevated amount of CD133 in their CSF $[321 \% \pm 210 \%(S D)$ or $58 \%(S E M) ; p=0.008]$ (Figure 2). Regression analysis revealed no association between CD133deviation and parameters of basic CSF-analysis (glucose, lactate, protein) (Figure 3).

\section{Subgroups of Chronic Inflammatory Disease}

Given that patients with chronic inflammatory diseases showed the highest increase in CSF-associated CD133 (Figure 2),

TABLE 1 | Clinical parameters of healthy subjects, patients with normal pressure hydrocephalus, parkinsonism, and dementia/cognitive impairment.

\begin{tabular}{ll}
\hline Parameters & \multicolumn{1}{c}{ Values } \\
\hline Healthy subjects $(n=10)$ & $53(49.3-61.5)$ \\
Age (years), median (range) & $6 / 4$ \\
Sex (female/male) & \\
Reason for lumbar puncture ( $n)$ & $2(20 \%)$ \\
$\quad$ Exclusion of subarachnoid hemorrhage & $8(80 \%)$ \\
$\quad$ Exclusion of inflammatory disease & \\
Normal pressure hydrocephalus ( $n=6)$ & $72(66-78)$ \\
Age (years), median (range) & $1 / 5$ \\
Sex (female/male) & \\
Parkinson syndrome ( $=6)$ & $73.0(68.5-78.5)$ \\
Age (years), median (range) & $4(66.67 \%)$ \\
Parkinson disease (\%) & $2(33.34 \%)$ \\
Atypical parkinsonism (\%) & \\
Dementia / cognitive impairment $(n=8)$ & $70.4(68.5-75.0)$ \\
Age (years), median (range) & $4 / 4$ \\
Sex (female/male) & $821(687-1232)$ \\
B-Amyloid 1-42 pg/ml (range) & $0.037(0.031-0.251)$ \\
Ratio B-Amyloid 1-42/1-40 & $486.5(358.0-749.5)$ \\
Total tau pg/ml (range) & $76.6-94.0)$ \\
Phosphor tau pg/ml (range) & \\
& \\
\hline & \\
\hline
\end{tabular}

TABLE 2 | Clinical parameters of the included patients with chronic inflammatory disease.

\begin{tabular}{|c|c|}
\hline Parameters & Values \\
\hline \multicolumn{2}{|c|}{ Subjects with central nervous inflammatory disease $(n=25)$} \\
\hline Age (years), median (range) & $47.5(41.25-53.75)$ \\
\hline Sex (female/male) & $(17 / 8)$ \\
\hline CIS / RRMS n (\%) & $7(28.0 \%)$ \\
\hline SPMS n (\%) & $10(40.0 \%)$ \\
\hline PPMS $n(\%)$ & $5(20.0 \%)$ \\
\hline Myelitis $n(\%)$ & $3(12.0 \%)$ \\
\hline Current EDSS score median (range) & $5.0(2.0-6.5)$ \\
\hline Disease duration (months), median (range) & $120(27-249)$ \\
\hline \multicolumn{2}{|l|}{ Neuroradiological data $(n=25)$} \\
\hline \multicolumn{2}{|l|}{ T2 lesions } \\
\hline \multicolumn{2}{|l|}{ Periventricular } \\
\hline Lesion 6-10 mm (\%) & $62.5 \%(15)$ \\
\hline Lesion > 10 mm (\%) & $50.0 \%(12)$ \\
\hline \multicolumn{2}{|l|}{ Juxtacortical } \\
\hline Lesion 6-10 mm (\%) & $66.7 \%(16)$ \\
\hline Lesion > 10 mm (\%) & $25.0 \%(6)$ \\
\hline \multicolumn{2}{|l|}{ Infratentorial } \\
\hline Lesion 6-10 mm (\%) & $29.1 \%(7)$ \\
\hline Lesion > 10 mm (\%) & $16.7 \%(4)$ \\
\hline Score (range) & $13.3(8-21)$ \\
\hline Spinal T2 lesions $(n=17)$ & $76.5 \%(13)$ \\
\hline \multicolumn{2}{|l|}{ T1 CE lesions } \\
\hline Cerebral lesions & $16.7 \%(4)$ \\
\hline Spinal lesions & $8.3 \%(2)$ \\
\hline \multicolumn{2}{|l|}{ Cerebral atrophy } \\
\hline Bicaudate ratio & $0.12(0.098-0.135)$ \\
\hline
\end{tabular}

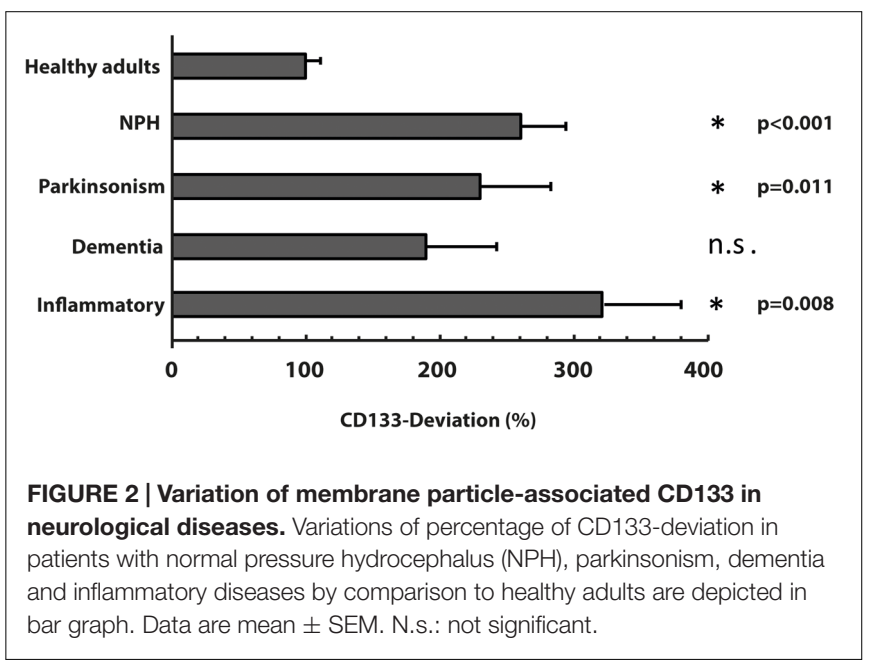

we performed a sub-analysis and grouped these 25 patients into relapsing-remitting multiple sclerosis (RRMS, including CIS; $n=7$ ), secondary progressive multiple sclerosis (SPMS, $n=10$ ), primary progressive multiple sclerosis (PPMS, $n=5)$ and patients with isolated myelitis $(n=3)$. Baseline characteristics, clinical and radiological parameters are shown in Table 2. 


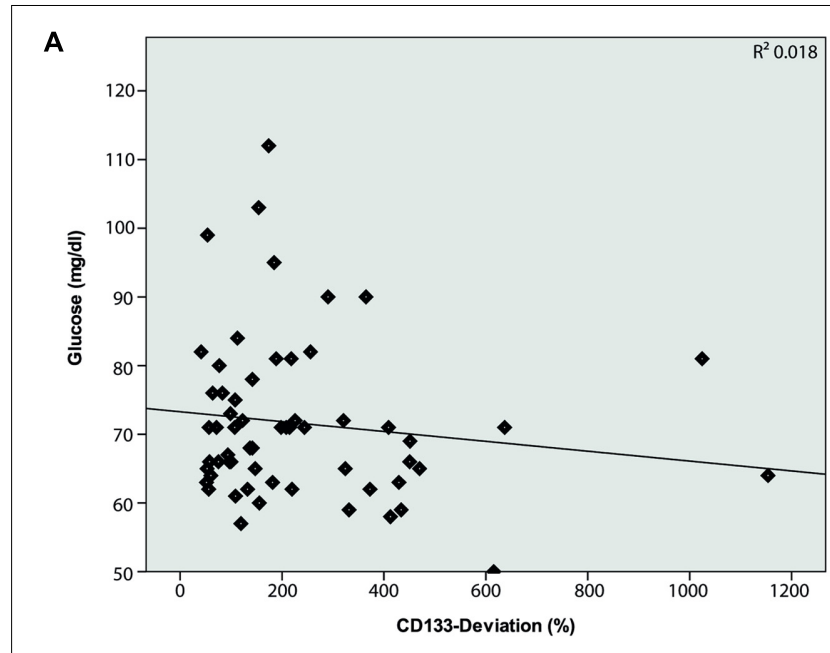

B

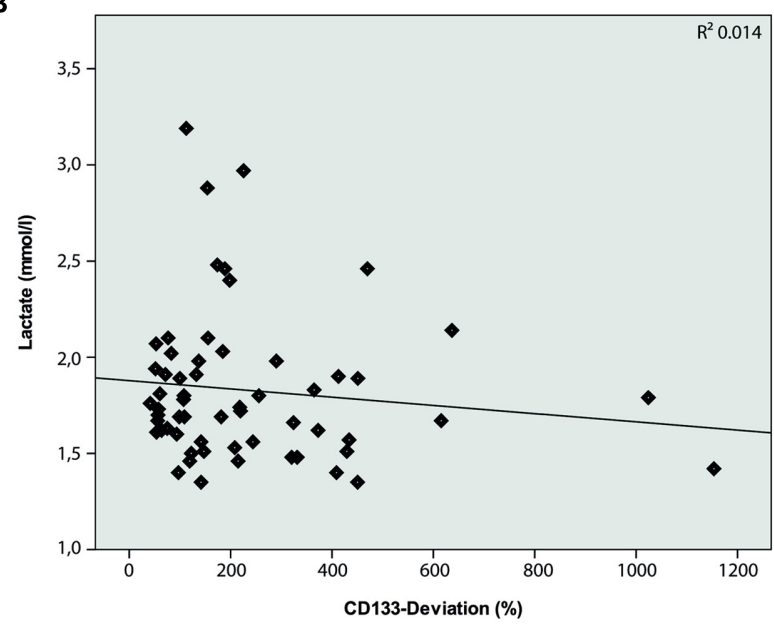

C

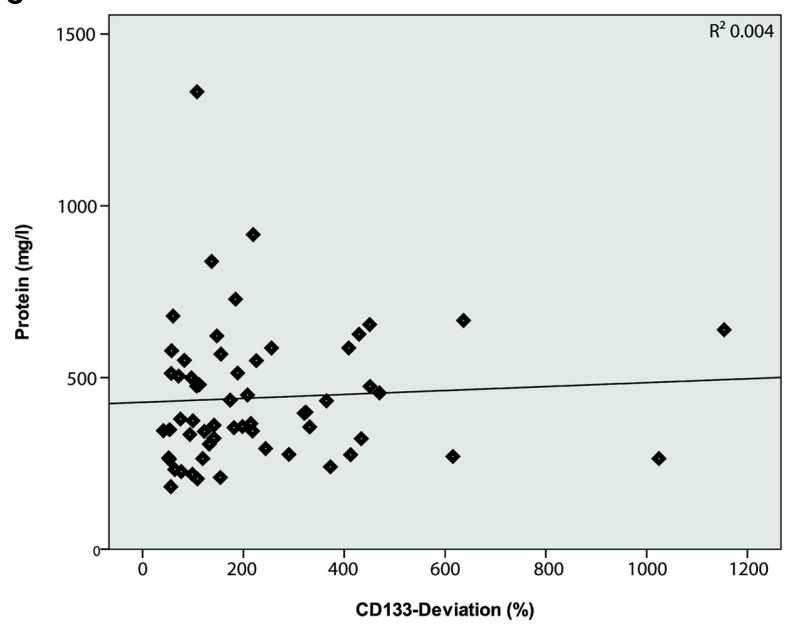

FIGURE 3 | Logistic regression model between CD133-deviation (\%) and basic CSF-analysis. Logistic regression model comparing CD133-deviation (\%) and Glucose $\left(\mathbf{A} ; R^{2}=0.018 ; p=0.312\right)$, Lactate (B; $\left.R^{2}=0.014 ; p=0.365\right)$, and Protein $\left(\mathbf{C} ; R^{2}=0.004 ; p=0.643\right)$. No correlation is found between these parameters.
Compared to healthy controls, levels of CD133 immunoreactivity in CSF revealed no increase in PPMS and myelitis-patients $[155 \% \pm 90 \%(S D)$ or $59 \%(\mathrm{SEM}) ; p=0.768$; and $222 \% \pm 134 \%(S D)$ or $109 \%$ (SEM); $p=0.273$, see Figure 4 ]. In contrast, patients with RRMS and SPMS showed increased CD133-levels compared to healthy controls $[291 \% \pm 91 \%(S D)$ or $76 \%(\mathrm{SEM}) ; p=0.023]$, and $434 \% \pm 135 \%(S D)$ or $111 \%$ (SEM); $p=0.010$, respectively. Finally, further analysis revealed no correlation between radiological parameters (lesion load / T2 lesions / T1 CE lesions / cerebral atrophy) $\left[R^{2}=0.019, p=0.513\right]$ and CD133-deviation, however, a statistical trend was seen when comparing EDSS-score with CD133 $\left(R^{2}=0.146, p=0.059\right.$, see Figure 5).

\section{DISCUSSION}

The present study for the first time systematically investigated human CSF in inflammatory and degenerative diseases for membrane particles carrying CD133. In essence, patients with parkinsonism, normal pressure hydrocephalus as well as with relapsing-remitting multiple sclerosis and SP multiple sclerosis showed markedly increased CD133 levels. Several aspects emerge from the data.

On a general note, analysis of membrane particles within the human CSF - compared to solely protein-based CSF investigation - may offer new approaches to study CNS diseases. CD133-positive membrane particles occur in CSF most likely as ectosomes, i.e., membrane particles derived from plasmalemma notably membrane protrusions. Specific CNS cells, such as ependymal cells and subventricular zone astrocytes which extend their cilium into the lumen, represent a plausible source of these CD133-positive membrane particles (Marzesco et al., 2005; Dubreuil et al., 2007; Corbeil et al., 2009, 2013; Kriegstein and Alvarez-Buylla, 2009). At the current stage of investigation, we cannot rule out the contribution of infiltration cells in the CSFassociated $\mathrm{CD} 133$ production. In this regard it has to be kept in

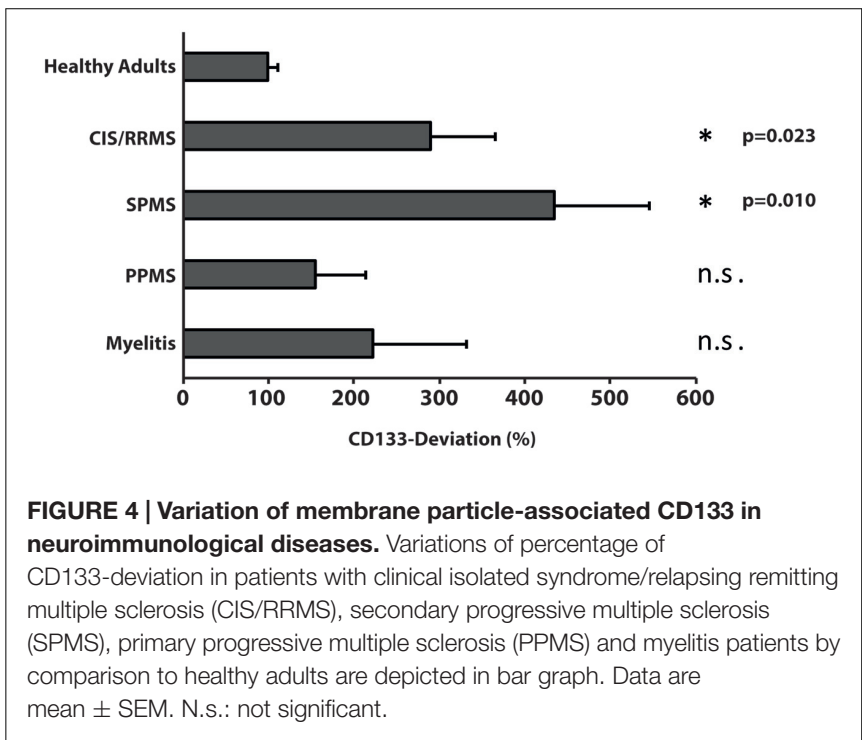




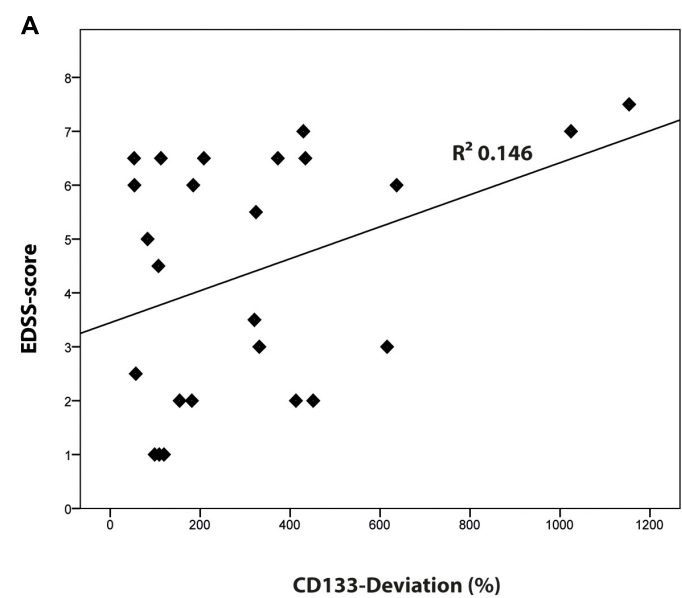

B

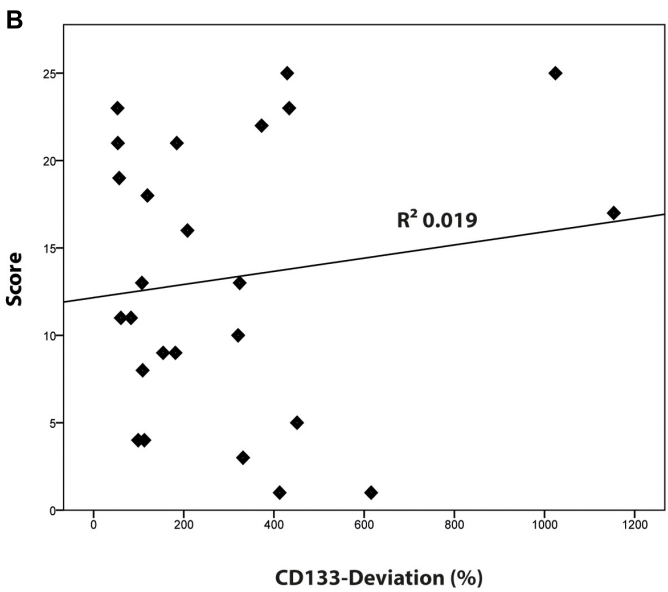

FIGURE 5 | Correlation of CD133 with clinical [Expanded Disability Status Scale (EDSS)-score] and radiological findings (lesion score). (A) Logistic regression model comparing the percentage of CD133-deviation and EDSS score $\left(R^{2}=0.146 ; p=0.059\right)$. (B) Logistic regression model comparing the percentage of CD133-deviation and the radiological lesion score $\left(R^{2}=0.019 ; p=0.513\right)$.

mind that our study did not reveal a correlation between basic CSF parameters like glucose, lactate, protein and CD133.

CD133 is also expressed by endothelial progenitor cells (Peichev et al., 2000). The diseases analyzed in this study may display processes of neovasculogenesis (Muramatsu et al., 2012) and/or blood-brain barrier dysfunction (Stolp and Dziegielewska, 2009). The presence of CD133 in myelin should not be neglected as well. Damage or remodeling of myelin sheaths might also contribute to the release of CD133-positive membrane particles by glia cells. In several diseases, a contribution of various cellular types and processes might account for the elevation of CD133 in CSF.

Given the fact that CD133 is associated to these CSF membrane particles, it has important clinical implications of a membrane particle-based CSF analysis for several reasons. Astrocytes and especially their reduced astrocytic response have been linked to neurodegenerative disease such as Alzheimer's disease and amyotrophic lateral sclerosis (Li et al., 2016). Results suggest that astrocytes affect oligodendrocytes via $A ß$ pathways in Alzheimer's disease, moreover, they are linked to progressive loss of corticospinal and spinal motor neurons by progressive reactive astrogliosis and reduction in myelin (Kang et al., 2013; Fu et al., 2015; Li et al., 2016). As shown here, also Parkinson's disease patients show increased CSF-associated CD133. Though, it remains speculative, if degeneration of myelinated axons from the substantia nigra may contribute to elevated levels of myelinderived CD133. As patients in the different groups differ by age, this raises the question of an additional influence of age on membrane particle associated CD133. However, our previous study demonstrated, that the concentration of CD133 is constant over age and this fact could not account for elevated levels of CD133 in several groups (Huttner et al., 2008).

Specifically interesting is the finding of increased CD133 in multiple sclerosis, as CD133 was found in myelin sheaths of glial cells (Corbeil et al., 2009). In multiple sclerosis, proinflammatory cytokines are assumed to attack pathogenic cells and destroy oligodendrocytes, myelin, and axons. Then protective trophic factors may modify the blood-brain barrier and modulate the extracellular matrix (Nait-Oumesmar et al., 2008; Guo et al., 2011; Kummerfeld et al., 2012; Cristofanilli et al., 2014; Li et al., 2016; Ludwin et al., 2016). Reactive gliogenesis on the one hand, massive myelin destruction with the release of myelin-derived CD133 into CSF on the other hand, may explain the high CD133-levels in multiple sclerosis, especially in those patients with a very high disease activity. It remains to be determined whether the inflammation process itself stimulates the release of CD133 and/or activates its expression. Interestingly, our analysis demonstrated a statistical trend for an elevation of CD133 in higher EDSS-scores. A relation between the inflammation and CD133 expression was noted in solid cancers (Karbanová et al., 2014; Rappa et al., 2015). Altogether, CD133 may serve as a surrogate marker for monitoring disease activity - similar to radiological findings - after initiation of immunomodulatory therapy (Harris and Sadiq, 2014; Khan et al., 2016; Uher et al., 2016; Zivadinov et al., 2016).

There are some shortcomings of the present study, notably the lack of repeated CSF analyses for longitudinal CD133 assessment. Moreover, findings would be more robust if they are replicated in a larger cohort of independent patients. Yet, results may have been biased by analysis of semi-quantitative immunoblotting, instead of ELISA or stronger methodological approaches, leaving room for some technical uncertainty and reliability. Finally, to determine the exact cellular source(s) of the membrane particles and their origin (plasma membrane versus multivesicular bodies), we would need an exhaustive characterization (e.g., proteomics and/or lipidomics) of CD133positive membrane particles isolated from each patient group (Redzic et al., 2014). These additional lines of investigation can extend our observation to other biomarkers, e.g., non-coding RNAs, and reveal discrete differences in the composition of membrane particles in various neurological disease (Tietje et al., 2014). So far, it remains unclear, if the isolated membrane particles are solely ectosomes or mixture of them with exosomes. Labeling for exosomal markers in future studies may solve this issue. Taken together, our results not only indicate a possible 
clinical value (e.g., biomarker for monitoring of several diseases), but also emphasize the need for further studies addressing all these shortcomings.

\section{CONCLUSION}

Our study revealed elevated levels of membrane particleassociated CD133 in patients with normal pressure hydrocephalus, parkinsonism, relapsing-remitting and secondary-progressive multiple sclerosis. Thus, CD133 may be of clinical value for several neurological diseases.

\section{AVAILABILITY OF DATA AND MATERIAL}

All data supporting this study are included in this published article and its supplementary information files.

\section{ETHICS STATEMENT}

The study was approved by the local institutional review board and informed consent was obtained from the participant.

\section{AUTHOR CONTRIBUTIONS}

TB: study concept, data acquisition, analysis and interpretation of data, writing the manuscript. LM: data acquisition, analysis and interpretation of data. HL: data acquisition, analysis and

\section{REFERENCES}

Bauer, N., Wilsch-Bräuninger, M., Karbanová, J., Fonseca, A. V., Strauss, D., Freund, D., et al. (2011). Haematopoietic stem cell differentiation promotes the release of prominin-1/CD133-containing membrane vesicles-a role of the endocytic-exocytic pathway. EMBO Mol. Med. 3, 398-409. doi: 10.1002/emmm. 201100147

Bermel, R. A., and Bakshi, R. (2006). The measurement and clinical relevance of brain atrophy in multiple sclerosis. Lancet Neurol. 5, 158-170. doi: 10.1016/ S1474-4422(06)70349-0

Chiasserini, D., Van Weering, J. R., Piersma, S. R., Pham, T. V., Malekzadeh, A., Teunissen, C. E., et al. (2014). Proteomic analysis of cerebrospinal fluid extracellular vesicles: a comprehensive dataset. J. Proteomics 106, 191-204. doi: 10.1016/j.jprot.2014.04.028

Codega, P., Silva-Vargas, V., Paul, A., Maldonado-Soto, A. R., Deleo, A. M., Pastrana, E., et al. (2014). Prospective identification and purification of quiescent adult neural stem cells from their in vivo niche. Neuron 82, 545-559. doi: 10.1016/j.neuron.2014.02.039

Colombo, E., Borgiani, B., Verderio, C., and Furlan, R. (2012). Microvesicles: novel biomarkers for neurological disorders. Front. Physiol. 3:63. doi: 10.3389/fphys. 2012.00063

Corbeil, D., Fargeas, C. A., and Jászai, J. (2014). CD133 might be a pan marker of epithelial cells with dedifferentiation capacity. Proc. Natl. Acad. Sci. U.S.A. 111, E1451-E1452. doi: 10.1073/pnas.1400195111

Corbeil, D., Joester, A., Fargeas, C. A., Jászai, J., Garwood, J., Hellwig, A., et al. (2009). Expression of distinct splice variants of the stem cell marker prominin-1 (CD133) in glial cells. Glia 57, 860-874. doi: 10.1002/glia. 20812 interpretation of data, critical revision of manuscript. SK: data acquisition, analysis and interpretation of data, critical revision of manuscript. PB: data acquisition, interpretation of data, critical revision of manuscript. PS: data acquisition, interpretation of data, critical revision of manuscript. JM: data acquisition, interpretation of data, critical revision of manuscript. DC: data acquisition, interpretation of data, critical revision of manuscript. $\mathrm{HH}$ : study concept, data acquisition, analysis and interpretation of data, writing and revision of manuscript.

\section{FUNDING}

This research did not receive any specific grant from funding agencies in the public, commercial, or not-for-profit sectors.

\section{ACKNOWLEDGMENTS}

The authors would like to thank Ulrike Naumann for her excellent technical assistance. We acknowledge support by Deutsche Forschungsgemeinschaft and Friedrich-AlexanderUniversität Erlangen-Nürnberg (FAU) within the funding programme Open Access Publishing.

\section{SUPPLEMENTARY MATERIAL}

The Supplementary Material for this article can be found online at: http://journal.frontiersin.org/article/10.3389/fncel. 2017.00077/full\#supplementary-material

Corbeil, D., Karbanová, J., Fargeas, C. A., and Jászai, J. (2013). Prominin-1 (CD133): molecular and cellular features across species. Adv. Exp. Med. Biol. 777, 3-24. doi: 10.1007/978-1-4614-5894-4_1

Corbeil, D., Röper, K., Hellwig, A., Tavian, M., Miraglia, S., Watt, S. M., et al. (2000). The human AC133 hematopoietic stem cell antigen is also expressed in epithelial cells and targeted to plasma membrane protrusions. J. Biol. Chem. 275, 5512-5520. doi: 10.1074/jbc.275.8. 5512

Coskun, V., Wu, H., Blanchi, B., Tsao, S., Kim, K., Zhao, J., et al. (2008). $\mathrm{CD} 133+$ neural stem cells in the ependyma of mammalian postnatal forebrain. Proc. Natl. Acad. Sci. U.S.A. 105, 1026-1031. doi: 10.1073/pnas.07100 00105

Cristofanilli, M., Rosenthal, H., Cymring, B., Gratch, D., Pagano, B., Xie, B., et al. (2014). Progressive multiple sclerosis cerebrospinal fluid induces inflammatory demyelination, axonal loss, and astrogliosis in mice. Exp. Neurol. 261, 620-632. doi: 10.1016/j.expneurol.2014.07.020

Dubreuil, V., Marzesco, A. M., Corbeil, D., Huttner, W. B., and WilschBräuninger, M. (2007). Midbody and primary cilium of neural progenitors release extracellular membrane particles enriched in the stem cell marker prominin-1. J. Cell Biol. 176, 483-495. doi: 10.1083/jcb.20060 8137

Fevrier, B., and Raposo, G. (2004). Exosomes: endosomal-derived vesicles shipping extracellular messages. Curr. Opin. Cell Biol. 16, 415-421. doi: 10.1016/j.ceb. 2004.06.003

Fu, W., Shi, D., Westaway, D., and Jhamandas, J. H. (2015). Bioenergetic mechanisms in astrocytes may contribute to amyloid plaque deposition and toxicity. J. Biol. Chem. 290, 12504-12513. doi: 10.1074/jbc.M114. 618157 
Grosse-Gehling, P., Fargeas, C. A., Dittfeld, C., Garbe, Y., Alison, M. R., Corbeil, D., et al. (2013). CD133 as a biomarker for putative cancer stem cells in solid tumours: limitations, problems and challenges. J. Pathol. 229, 355-378. doi: 10.1002/path.4086

Guo, F., Maeda, Y., Ma, J., Delgado, M., Sohn, J., Miers, L., et al. (2011). Macroglial plasticity and the origins of reactive astroglia in experimental autoimmune encephalomyelitis. J. Neurosci. 31, 11914-11928. doi: 10.1523/JNEUROSCI. 1759-11.2011

Harris, V. K., and Sadiq, S. A. (2014). Biomarkers of therapeutic response in multiple sclerosis: current status. Mol. Diagn. Ther. 18, 605-617. doi: 10.1007/ s40291-014-0117-0

Hühmer, A. F., Biringer, R. G., Amato, H., Fonteh, A. N., and Harrington, M. G. (2006). Protein analysis in human cerebrospinal fluid: physiological aspects, current progress and future challenges. Dis. Markers 22, 3-26. doi: 10.1155/ 2006/158797

Huttner, H. B., Corbeil, D., Thirmeyer, C., Coras, R., Köhrmann, M., Mauer, C., et al. (2012). Increased membrane shedding-indicated by an elevation of CD133-enriched membrane particles-into the CSF in partial epilepsy. Epilepsy Res. 99, 101-106. doi: 10.1016/j.eplepsyres.2011.10.029

Huttner, H. B., Janich, P., Köhrmann, M., Jászai, J., Siebzehnrubl, F., Blümcke, I., et al. (2008). The stem cell marker prominin-1/CD133 on membrane particles in human cerebrospinal fluid offers novel approaches for studying central nervous system disease. Stem Cells 26, 698-705. doi: 10.1634/stemcells.20070639

Jászai, J., Graupner, S., Tanaka, E. M., Funk, R. H., Huttner, W. B., Brand, M., et al. (2013). Spatial distribution of prominin-1 (CD133)-positive cells within germinative zones of the vertebrate brain. PLOS ONE 8:e63457. doi: 10.1371/ journal.pone.0063457

Johanson, C., Stopa, E., Mcmillan, P., Roth, D., Funk, J., and Krinke, G. (2011). The distributional nexus of choroid plexus to cerebrospinal fluid, ependyma and brain: toxicologic/pathologic phenomena, periventricular destabilization, and lesion spread. Toxicol. Pathol. 39, 186-212. doi: 10.1177/019262331039 4214

Kang, S. H., Li, Y., Fukaya, M., Lorenzini, I., Cleveland, D. W., Ostrow, L. W., et al. (2013). Degeneration and impaired regeneration of gray matter oligodendrocytes in amyotrophic lateral sclerosis. Nat. Neurosci. 16, 571-579. doi: 10.1038/nn.3357

Karbanová, J., Laco, J., Marzesco, A. M., Janich, P., Voborníková, M., Mokrý, J., et al. (2014). Human prominin-1 (CD133) is detected in both neoplastic and non-neoplastic salivary gland diseases and released into saliva in a ubiquitinated form. PLoS ONE 9:e98927. doi: 10.1371/journal.pone.0098927

Karbanová, J., Missol-Kolka, E., Fonseca, A. V., Lorra, C., Janich, P., Hollerová, H., et al. (2008). The stem cell marker CD133 (Prominin-1) is expressed in various human glandular epithelia. J. Histochem. Cytochem. 56, 977-993. doi: 10.1369/ jhc.2008.951897

Khan, O., Seraji-Bozorgzad, N., Bao, F., Razmjou, S., Caon, C., Santiago, C., et al. (2016). The relationship between brain MR spectroscopy and disability in multiple sclerosis: 20 -year data from the U.S. glatiramer acetate extension study. J. Neuroimaging 27, 97-106. doi: 10.1111/jon.12358

Kriegstein, A., and Alvarez-Buylla, A. (2009). The glial nature of embryonic and adult neural stem cells. Annu. Rev. Neurosci. 32, 149-184. doi: 10.1146/annurev. neuro.051508.135600

Kummerfeld, M., Seehusen, F., Klein, S., Ulrich, R., Kreutzer, R., Gerhauser, I., et al. (2012). Periventricular demyelination and axonal pathology is associated with subependymal virus spread in a murine model for multiple sclerosis. Intervirology 55, 401-416. doi: 10.1159/000336563

Kurtzke, J. F. (1983). Rating neurologic impairment in multiple sclerosis: an expanded disability status scale (EDSS). Neurology 33, 1444-1452. doi: 10.1212/ WNL.33.11.1444

Li, J., Zhang, L., Chu, Y., Namaka, M., Deng, B., Kong, J., et al. (2016). Astrocytes in oligodendrocyte lineage development and white matter pathology. Front. Cell Neurosci. 10:119. doi: 10.3389/fncel.2016.00119

Ludwin, S. K., Rao, V. T., Moore, C. S., and Antel, J. P. (2016). Astrocytes in multiple sclerosis. Mult. Scler. 22, 1114-1124. doi: 10.1177/135245851664 3396

Marzesco, A. M., Janich, P., Wilsch-Bräuninger, M., Dubreuil, V., Langenfeld, K., Corbeil, D., et al. (2005). Release of extracellular membrane particles carrying the stem cell marker prominin-1 (CD133) from neural progenitors and other epithelial cells. J. Cell Sci. 118, 2849-2858. doi: 10.1242/jcs.02439

Miraglia, S., Godfrey, W., Yin, A. H., Atkins, K., Warnke, R., Holden, J. T., et al. (1997). A novel five-transmembrane hematopoietic stem cell antigen: isolation, characterization, and molecular cloning. Blood 90, 5013-5021.

Montalban, X., Tintore, M., Swanton, J., Barkhof, F., Fazekas, F., Filippi, M., et al. (2010). MRI criteria for MS in patients with clinically isolated syndromes. Neurology 74, 427-434. doi: 10.1212/WNL.0b013e3181cec45c

Muramatsu, R., Takahashi, C., Miyake, S., Fujimura, H., Mochizuki, H., and Yamashita, T. (2012). Angiogenesis induced by CNS inflammation promotes neuronal remodeling through vessel-derived prostacyclin. Nat. Med. 18, 1658-1664. doi: 10.1038/nm.2943

Nait-Oumesmar, B., Picard-Riéra, N., Kerninon, C., and Baron-Van Evercooren, A. (2008). The role of SVZ-derived neural precursors in demyelinating diseases: from animal models to multiple sclerosis. J. Neurol. Sci. 265, 26-31. doi: 10.1016/j.jns.2007.09.032

Nogueira, A. B., Sogayar, M. C., Colquhoun, A., Siqueira, S. A., Nogueira, A. B., Marchiori, P. E., et al. (2014). Existence of a potential neurogenic system in the adult human brain. J. Transl. Med. 12:75. doi: 10.1186/1479-5876$12-75$

Ormerod, I. E., Miller, D. H., Mcdonald, W. I., Du Boulay, E. P., Rudge, P., Kendall, B. E., et al. (1987). The role of NMR imaging in the assessment of multiple sclerosis and isolated neurological lesions. A quantitative study. Brain 110(Pt 6), 1579-1616. doi: 10.1093/brain/110.6.1579

Peichev, M., Naiyer, A. J., Pereira, D., Zhu, Z., Lane, W. J., Williams, M., et al. (2000). Expression of VEGFR-2 and AC133 by circulating human CD34(+) cells identifies a population of functional endothelial precursors. Blood 95, 952-958.

Petzold, A., Steenwijk, M. D., Eikelenboom, J. M., Wattjes, M. P., and Uitdehaag, B. M. (2016). Elevated CSF neurofilament proteins predict brain atrophy: a 15-year follow-up study. Mult. Scler. 22, 1154-1162. doi: 10.1177/ 1352458516645206

Pruszak, J. (2015). Neural Surface Antigens: From Basic Biology Towards Biomedical Applications. London: Elsevier.

Rappa, G., Fargeas, C. A., Le, T. T., Corbeil, D., and Lorico, A. (2015). Letter to the editor: an intriguing relationship between lipid droplets, cholesterol-binding protein $\mathrm{CD} 133$ and Wnt/beta-catenin signaling pathway in carcinogenesis. Stem Cells 33, 1366-1370. doi: 10.1002/stem.1953

Redzic, J. S., Ung, T. H., and Graner, M. W. (2014). Glioblastoma extracellular vesicles: reservoirs of potential biomarkers. Pharmgenomics Pers. Med. 7, 65-77. doi: 10.2147/PGPM.S39768

Rovira, A., Swanton, J., Tintore, M., Huerga, E., Barkhof, F., Filippi, M., et al. (2009). A single, early magnetic resonance imaging study in the diagnosis of multiple sclerosis. Arch. Neurol. 66, 587-592. doi: 10.1001/archneurol.2009.49

Stolp, H. B., and Dziegielewska, K. M. (2009). Review: role of developmental inflammation and blood-brain barrier dysfunction in neurodevelopmental and neurodegenerative diseases. Neuropathol. Appl. Neurobiol. 35, 132-146.doi: 10. $1111 /$ j.1365-2990.2008.01005.x

Tietje, A., Maron, K. N., Wei, Y., and Feliciano, D. M. (2014). Cerebrospinal fluid extracellular vesicles undergo age dependent declines and contain known and novel non-coding RNAs. PLOS ONE 9:e113116. doi: 10.1371/journal.pone. 0113116

Uher, T., Vaneckova, M., Sobisek, L., Tyblova, M., Seidl, Z., Krasensky, J., et al. (2016). Combining clinical and magnetic resonance imaging markers enhances prediction of 12-year disability in multiple sclerosis. Mult. Scler. 23, 51-61. doi: $10.1177 / 1352458516642314$

Walker, T. L., Wierick, A., Sykes, A. M., Waldau, B., Corbeil, D., Carmeliet, P., et al. (2013). Prominin-1 allows prospective isolation of neural stem cells from the adult murine hippocampus. J. Neurosci. 33, 3010-3024. doi: 10.1523/ JNEUROSCI.3363-12.2013

Weigmann, A., Corbeil, D., Hellwig, A., and Huttner, W. B. (1997). Prominin, a novel microvilli-specific polytopic membrane protein of the apical surface of epithelial cells, is targeted to plasmalemmal protrusions of non-epithelial cells. Proc. Natl. Acad. Sci. U.S.A. 94, 12425-12430. doi: 10.1073/pnas.94.23. 12425

Zetterberg, H. (2015). Cerebrospinal fluid biomarkers for Alzheimer's disease: current limitations and recent developments. Curr. Opin. Psychiatry 28, 402-409. doi: 10.1097/YCO.0000000000000179 
Zivadinov, R., Uher, T., Hagemeier, J., Vaneckova, M., Ramasamy, D. P., Tyblova, M., et al. (2016). A serial 10-year follow-up study of brain atrophy and disability progression in RRMS patients. Mult. Scler. 22, 1709-1718. doi: $10.1177 / 1352458516629769$

Conflict of Interest Statement: The authors declare that the research was conducted in the absence of any commercial or financial relationships that could be construed as a potential conflict of interest.
Copyright @ 2017 Bobinger, May, Lücking, Kloska, Burkardt, Spitzer, Maler, Corbeil and Huttner. This is an open-access article distributed under the terms of the Creative Commons Attribution License (CC BY). The use, distribution or reproduction in other forums is permitted, provided the original author(s) or licensor are credited and that the original publication in this journal is cited, in accordance with accepted academic practice. No use, distribution or reproduction is permitted which does not comply with these terms. 